\title{
Navegação em software livre e impressão 3D: princípios básicos e simulações em
}

\section{Cirurgia e Traumatologia Buco-Maxilo-Faciais}

\author{
Free software navigation and 3D printing: basic principles and simulations in Oral and \\ Maxillofacial Surgery \\ Navegación de software libre e impresión 3D: principios básicos y simulaciones en Cirugía Oral y \\ Maxilofacial
}

Alexandre Silva de Quevedo

ORCID: https://orcid.org/0000-0001-5613-8015 Universidade Federal do Rio Grande do Sul, Brasil

E-mail: quevedoalexandre@hotmail.com

Deise Ponzoni

ORCID: https://orcid.org/0000-0003-2855-7495

Hospital de Clínicas de Porto Alegre, Brasil Universidade Federal do Rio Grande do Sul, Brasil E-mail: deise.ponzoni@ufrgs.br

\begin{abstract}
Resumo
A utilização de sistemas computacionais permite a educação continuada aos profissionais, o estudo e planejamento cirúrgico virtual e a obtenção de objetos físicos diretamente a partir dos dados gerados. As imagens obtidas a partir da tomografia computadorizada podem ser manipuladas através de softwares livres. A prototipagem rápida é amplamente utilizada em Cirurgia e Traumatologia Buco-maxilo-faciais permitindo aumentar a previsibilidade cirúrgica, reduzindo o tempo associado ao procedimento e consequentemente resultando em menor morbidade ao paciente, baixando os custos vinculados à assistência em saúde. Este trabalho descreve o protocolo do emprego de técnicas de prototipagem rápida aplicadas a pacientes com necessidades de tratamento na especialidade de Cirurgia e Traumatologia Buco-maxilo-faciais, em nível terciário de atenção em saúde, vinculados ao Sistema Único de Saúde, em um hospital escola do sul do Brasil. Explora ainda, parâmetros relacionados a aquisição da imagem por tomografia computadorizada, características estruturais dos softwares utilizados, dos equipamentos e critérios de qualidade dimensional dos materiais utilizados. A adoção dessas tecnologias poderá trazer melhora na qualidade assistencial, devendo ser estimulada dentro do Sistema Único de Saúde.
\end{abstract}

Palavras-chave: Impressão 3D; Realidade virtual; Cirurgia bucal.

\section{Abstract}

The use of computer systems allows professionals to continue education, study and virtual surgical planning, and obtain physical objects directly from the generated data. The images obtained from computed tomography can be 
manipulated using free software. Rapid prototyping is widely used in Oral and Maxillofacial Surgery and Traumatology, allowing for increased surgical predictability, reducing the time associated with the procedure and consequently resulting in lower patient morbidity, lowering the costs associated with health care. This paper describes the protocol for the use of rapid prototyping techniques applied to patients with treatment needs in the specialty of Oral and Maxillofacial Surgery and Traumatology, at the tertiary level of health care, linked to the Unified Health System, in a teaching hospital from the south of Brazil. It also explores parameters related to image acquisition by computed tomography, structural characteristics of the software used, equipment and dimensional quality criteria of the materials used. The adoption of these technologies improves the quality of care and must be encouraged within the Unified Health System.

Keywords: Printing, Three-dimensional; Virtual reality; Surgery, oral.

\section{Resumen}

El uso de sistemas informáticos permite la formación continua de los profesionales, el estudio y planificación quirúrgica virtual y la adquisición de objetos físicos directamente a partir de los datos generados. Las imágenes obtenidas de la tomografía computarizada se pueden manipular mediante software gratuito. La creación rápida de prototipos se usa ampliamente en Cirugía y Traumatología Oral y Maxilofacial, lo que permite una mayor predictibilidad quirúrgica, reduce el tiempo asociado con el procedimiento y, en consecuencia, reduce la morbilidad del paciente, lo que reduce los costos asociados con la atención médica. En este artículo se describe el protocolo de uso de técnicas de prototipado rápido aplicadas a pacientes con necesidades de tratamiento en la especialidad de Cirugía Oral y Maxilofacial, en el nivel terciario de la atención de la salud, vinculado al Sistema Único de Salud, en un hospital docente del sur del Brasil. También explora parámetros relacionados con la adquisición de imágenes por tomografía computada, características estructurales del software utilizado, equipos y criterios de calidad dimensional de los materiales utilizados. La adopción de estas tecnologías mejora la calidad de la atención y debe fomentarse dentro del Sistema Único de Salud.

Palabras clave: Impresión tridimensional; Realidad virtual; Cirugía bucal.

\section{Introdução}

Diversas aplicações de tecnologias de navegação por softwares e impressão tridimensional foram introduzidas na área da saúde. Destacam-se o treinamento cirúrgico digital; confecção de biomodelos, para fins protéticos, educacionais e impressão de tecidos ou órgãos (Ganry et al., 2018; Ibrahim et al., 2009; Mehrotra \& Markus, 2021; Meurer et al., 2003; Stansbury \& Idacavage, 2016). Nessa perspectiva, muitas delas podem ser desenvolvidas e aplicadas para fins de diagnóstico e apoio ao tratamento aos usuários do Sistema Único de Saúde (SUS) na especialidade da Cirurgia e Traumatologia Bucomaxilo-faciais (CTBMF). Essa especialidade odontológica faz o diagnóstico e o tratamento cirúrgico e adjuvante de doenças, traumatismos, lesões congênitas ou adquiridas do sistema estomatognático e estruturas craniofaciais associadas (Puricelli, 2014).

A busca por procedimentos cirúrgicos de excelência, menos invasivos e rápidos proporcionou o desenvolvimento de planejamentos cirúrgicos digitais, conduzindo o cirurgião a evoluir tecnicamente, gerando diagnósticos mais apurados e refinando as áreas de interesse para estudos de casos de maior complexidade. Além disso, garantiu o treinamento continuado dos profissionais (Ganry et al., 2018; Mehrotra \& Markus, 2021). A imagem gerada e reconstruída por equipamentos de diagnósticos não invasivos como tomografia computadorizada (TC), ressonância magnética (RM) e equipamentos ultrassônicos permitem visualização apurada, anatomicamente correta e fidedigna das estruturas corpóreas (Lechuga \& Weidlich, 2016; Steenberghe et al., 2002). Ao definir a área da intervenção cirúrgica, o cirurgião pode realizar modelagem sólida das imagens, segmentar e aglutinar diferentes estruturas teciduais através de várias ferramentas disponibilizadas em softwares livres. Estas ferramentas dispostas em sistemas computacionais, com recursos auxiliares, viabilizam o planejamento pré-operatório individualizado e permitem a obtenção de objetos físicos diretamente a partir dos dados gerados (prototipagem rápida). A Cirurgia e Traumatologia Buco-maxilo-faciais é uma das especialidades da saúde que mais utilizam prototipagem rápida (PR) para confecção de modelos anatômicos e guias cirúrgicos (Calvo-Haro et al., 2021; Ganry et al., 2018; Mehrotra \& Markus, 2021; Meurer et al., 2008; Mitsouras et al., 2015). 
A evolução do planejamento cirúrgico tradicional para o planejamento cirúrgico digital, que permite a confecção de réplicas físicas, guias cirúrgicos ou próteses intraoperatórias através de modelos físicos tridimensionais, pelo método de manufatura aditiva exige o cumprimento de diferentes etapas.

Este trabalho descreve um protocolo para confecção de biomodelos, empregando técnicas de prototipagem rápida (PR) para tratamento de pacientes na especialidade de Cirurgia e Traumatologia Buco-maxilo-faciais, em um hospital escola do sul do país. Analisa ainda, parâmetros relacionados a aquisição da imagem por TC, características estruturais dos softwares utilizados, dos equipamentos de PR e critérios de qualidade dimensional dos materiais utilizados.

\section{Metodologia}

O processo descrito é o resultado do trabalho conjunto entre o Serviço de Radiologia, o Serviço de Pesquisa e Desenvolvimento em Engenharia Biomédica e a Unidade de Cirurgia Buco-maxilo-facial do Hospital de Clínicas de Porto Alegre (HCPA). Faz parte de um projeto de desenvolvimento para implementação de tecnologia junto ao atendimento de pacientes com necessidades de tratamento na especialidade de Cirurgia e Traumatologia Buco-maxilo-faciais, em nível terciário de atenção em saúde, vinculados ao SUS. O projeto foi aprovado pelo Comitê de Ética em Pesquisa do HCPA (número 2020-0410). Estão incluídos pacientes com deformidades dento-esqueléticas, lesões tumorais e sequelas de trauma panfacial, que resultem em importante deformidade e prejuízo funcional. O processo envolve três etapas distintas: a aquisição da geometria do segmento corporal, através de Tomografia Computadorizada de Feixe em Leque (TCFL) e geração de uma superfície 3D (Three Dimension); manipulação e modelagem do objeto 3D, com geração de um corpo sólido através de ferramentas CAD (Computer Aided Design) 3D, utilizando softwares livres e, por fim, a confecção de biomodelos, através do processo de modelagem por FFF (Fused Filament Fabrication), ou seu sinônimo FDM (Fused Deposition Modelling).

A TCFL apresenta menor presença de artefato, menos ruído, maior relação sinal-ruído e maior capacidade de discriminar estruturas anatômicas quando comparada com a Tomografia Computadorizada Cone Beam (TCCB). O sistema de TCCB é mais propenso à dispersão e endurecimento do feixe, resultando em imagens menos uniformes (Lechuga \& Weidlich, 2016). A TCFL é predominante nos serviços de imagem dos ambientes hospitalares.

\subsection{Aquisição e reconstrução de imagens adquiridas em tomógrafo hospitalar}

As imagens foram obtidas por um tomógrafo multislice Aquilion CX, 64 canais (Toshiba Medical Systems Corporation, Otawara, Japão) através do protocolo de aquisição das imagens para exame de região de cabeça e pescoço sugerido pelo fabricante. O paciente foi posicionado em decúbito dorsal com braços paralelos ao longo do corpo, visando a posição mais confortável possível com a utilização de almofadas temporais. Não foram utilizadas cinta para a cabeça, nem faixa para imobilização do mento. Foi utilizado suporte para alinhar a cabeça do paciente com a linha de referencia órbitomeatal (LOM) e foi utilizado feixe de luz superior sobre a metade do mento para que a imagem fosse adquirida simetricamente sobre o plano sagital. Utilizou-se energia para tensão de alimentação $128 \mathrm{kV}$, com espessura de corte de $3 \mathrm{~mm}$ e reconstrução das imagens em uma proporção de 1 para $0,8 \mathrm{~mm}$. O arquivo gerado foi salvo em formato DICOM (Digital Imagin and Comunications in Medicine), o que possibilitou a reconstrução tridimensional. Devido à complexidade da geometria e da resolução da TCFL foi necessário a formação de um algoritmo computacional (malha), que permitiu a identificação de falhas e reduziu a quantidade de ruídos aleatórios. Dessa forma, obteve-se uma superfície o mais regular possível, onde os artefatos indesejados foram removidos (Bagaria \& Chaudhary, 2017).

Para criação da malha 3D e manipulação dos modelos anatômicos virtuais foi utilizado o software 3D Slicer® (The Slicer Community, Harvard, MA, USA). A janela de densidade foi determinada, estabelecendo-se o intervalo dos níveis de 
cinza desejado (Escada de Hounsfield, sendo largura da janela de 2000HU e centro da janela 500HU), de acordo com a região de interesse (região de face, segmentação das imagens).

Após aquisição e segmentação da área de interesse foi possível converter o arquivo em formato DICOM para o formato STL (Standard Template Library) e subsequente envio para impressora 3D, ou converter o arquivo DICOM em um programa de modelagem sólida salvo em CAD-CAM (Computer-Aided Design - Computer-Aided Manufacturing), possibilitando ao profissional a manipulação, modelagem e/ou criação de estruturas sólidas sobre a imagem original, para realização de simulações operatórias.

\subsection{Manipulação e modelagem em ferramentas para imagens 3D}

Com o arquivo salvo no formato STL ou CAD-CAM, a modelagem e renderização das imagens foram realizadas com os softwares 3D Builder® (18.0.1931.0, Microsoft Corporation, Redmond, Washington, EUA) e Meshmixer® (v3.5, Autodesk, São Rafael, EUA), respectivamente. Nesses softwares, os objetos 3D são identificados por uma nuvem de pontos através de uma malha poligonal composta por um conjunto de triângulos ou quadriláteros interligados por seus vértices (Figuras 1 e 2), que em conjunto definem os limites do objeto 3D tridimensionalmente (largura, comprimento e profundidade) (Oliveira et al., 2014).

Figura 1: Imagem obtida a partir da utilização do software Meshmixer® (v3.5, Autodesk, São Rafael, EUA) na visualização de uma reconstrução 3D de uma TC hospitalar.

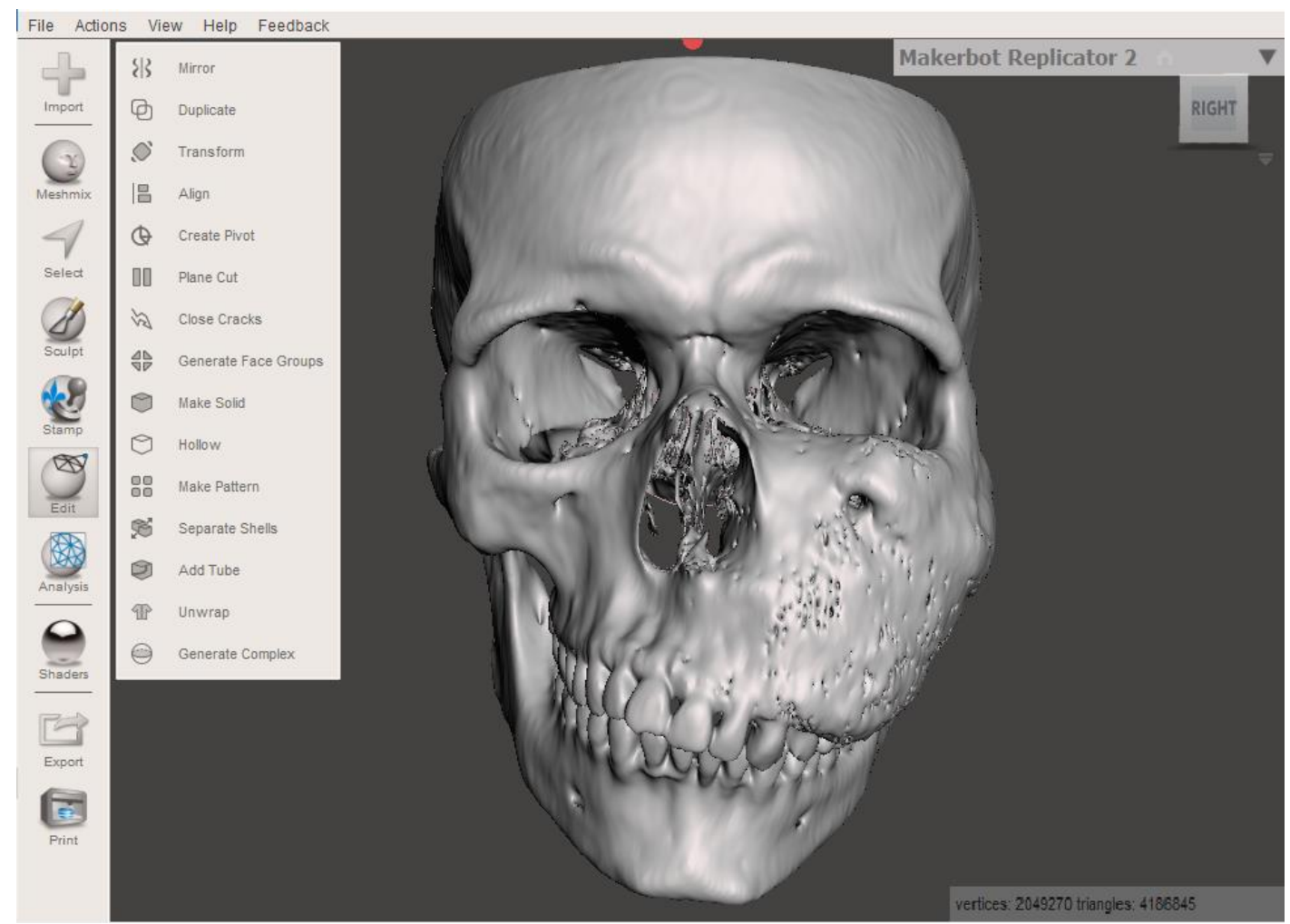

No quadro, à esquerda da imagem, observa-se a paleta de ferramentas disponíveis para manipulação da imagem. A reconstrução tridimensional mostra importante assimetria facial causada pela lesão, do lado esquerdo do paciente. Fonte: Autores. 
Figura 2: Segmentação e manipulação das estruturas ósseas realizadas pelo software 3d Builder® (18.0.1931.0, Microsoft Corporation, Redmond, Washington, EUA).

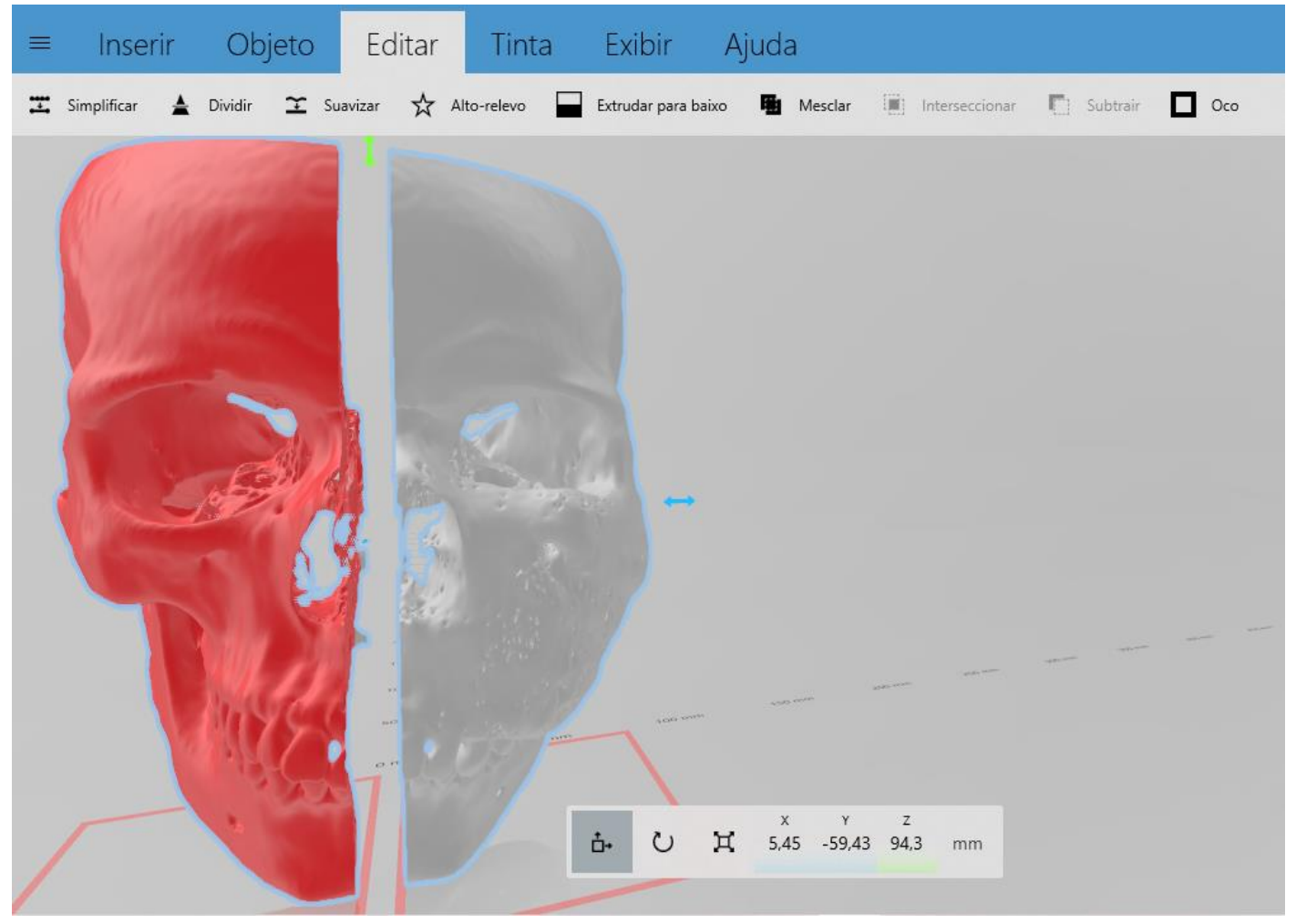

Na barra superior da imagem, observa-se a paleta de ferramentas disponíveis para manipulação da imagem. As capacidades de edição são diferentes e em menor número quando comparadas ao software Meshmixer®. Fonte: Autores.

Após a manipulação das imagens, exclusão, inclusão, criação de suportes e guias cirúrgicos as imagens manipuladas em CAD-CAM foram convertidas em formato STL, formato padrão para a maioria das impressoras 3D.

\subsection{Impressão 3D por manufatura aditiva}

Para a impressão, o objeto 3D foi exportado para o software Cura pertencente a Ultimaker (Versão 4.11.0, Ultimaker, Geldermalsen, Holland). Esse software permitiu o planejamento da impressão, proporcionando a manutenção da estabilidade dimensional e estrutural da peça.

A impressão dos biomodelos/guias foi realizada com as impressoras Ultimaker modelo 3 e Ultimaker modelo S5 (Ultimaker BV, Utrecht, Holland) através da técnica de modelagem por FFF. A matéria prima utilizada foi CPE+ (Chlorinated PolyEthene) termoplástico, em filamento. A confecção da peça ocorreu através de camadas incrementais, com resolução geométrica por camada de $0,1 \mathrm{~mm}$.

\section{Resultados e Discussão}

O estabelecimento de protocolo para a confecção de biomodelos/guias cirúrgicos empregando PR envolve diferentes etapas, que são sequenciais. A presença de equívocos em qualquer passo da criação poderá conduzir a um conjunto de erros que inviabilizam a apropriada criação do objeto 3D digital ou impresso (Attene et al., 2013; Ibrahim et al., 2009; Kim et al., 2018; Roça et al., 2013; Stansbury \& Idacavage, 2016).

A TC é uma importante ferramenta de diagnóstico, que utiliza radiação ionizante para a obtenção de imagens anatômicas tridimensionais com alta capacidade diagnóstica. Os exames tomográficos obtidos a partir de tomógrafos 
hospitalares, são amplamente utilizados em Cirurgia e Traumatologia Buco-maxilo-faciais, em atendimentos de pacientes vinculados ao SUS. No período de 2008 a 2011 foi registrado um aumento de 9\% na quantidade de tomógrafos computadorizados disponíveis no SUS, sendo a TC de face, o exame tomográfico mais solicitado (Dovales et al., 2016). No Serviço de Radiologia do Hospital de Clínicas de Porto Alegre são realizados, em média, 400 exames tomográficos de ossos da face por ano.

$\mathrm{Na}$ execução do exame, quanto menor a espessura dos cortes, maior o tempo de aquisição e, consequentemente, maior a dose de radiação ao qual os pacientes são expostos (Dalmazo et al., 2010). Por outro lado, quanto menor for a espessura dos cortes e os intervalos da reconstrução, com espaçamento isocúbico padronizado, melhor será a qualidade da imagem. A qualidade das peças impressas é diretamente dependente da aquisição e da qualidade de resolução da imagem (Meurer et al., 2008, Eijnatten et al., 2018). Cortes acima de $2 \mathrm{~mm}$ podem gerar baixa resolução e consideráveis distorções nas peças geradas a partir da reconstrução de imagens (Whyms et al., 2013). O protocolo de aquisição realizado seguiu a recomendação do fabricante, garantindo a minimização de exposição à radiação para o paciente e otimizando a qualidade da reconstrução das imagens 3D. Dessa forma, evitou-se a necessidade de repetição de exames e erros dimensionais da TC adquirida, com subsequente prejuízo à impressão do objeto.

A criação da malha 3D e a manipulação dos modelos anatômicos virtuais, com a utilização do software 3D Slicer®, é um processo operador-dependente e demandou do profissional amplo conhecimento da anatomia, de modo que fossem evitadas modificações na morfologia das estruturas. O profissional realizou treinamento para utilização do software. Contudo, não foi realizada a calibração. Uma parte desafiadora desta fase do processo de trabalho foi a determinação dos tecidos a serem analisados, selecionando a janela desejada. O nível de cinza dos pixels nos arquivos DICOM foi determinado pela densidade dos tecidos; a segmentação dos tecidos foi realizada de forma limiar, a fim de estabelecer a região de interesse e diminuir o trabalho de extração de partes que não eram úteis para a confecção da malha 3D (Jacobs et al., 2008; Li et al., 2015; Shui et al., 2017).

A prototipagem em realidade virtual, auxiliada por recursos de reconstruções virtuais permitiu ao profissional a manipulação do modelo computadorizado de forma muito próxima ao real, em verdadeira grandeza. Tal condição gerou a representação fiel da forma digital no produto final. Proporcionou ainda, a modelagem, interação, exploração de dados geométricos e densidades anatômicas (Volpato, 2007).

Os softwares Meshmixer® (Figura 1) e 3d Builder® (Figuras 2 e 3) permitiram o modelamento das imagens 3D. Ambos são ferramentas com licenças abertas (gratuitos) para uso, que apresentam funcionalidades para manipulação de imagens baseadas em malhas poligonais. A manipulação das imagens através dos softwares, não necessitou alto grau de treinamento prévio por parte do profissional, uma vez que as interfaces são claras e autoexplicativas (Meshmixern, 2021). A utilização de modelos poligonais, gerados a partir de imagens envolvendo a região buco-maxilo-facial, pode estar relacionada a erros dimensionais, uma vez que requerem um maior número de triângulos (faces) para reproduzir uma superfície mais real e detalhada. Quanto maior a quantidade de vértices e triângulos do arquivo 3D, maiores serão o tamanho e o tempo de processamento do arquivo (Figura 1) (Ju, 2009). Visando a manutenção da riqueza de detalhes das imagens, não foram utilizadas ferramentas de redução de complexidade da malha trabalhada. A exportação das imagens tomográficas, sua manipulação e modelagem em diferentes softwares e o arquivamento em diferentes formatos gerou repetitivos erros de armazenamento. Apesar dos softwares apresentarem boa resolução de imagem, são lentos e exigem grande capacidade computacional.

Os erros geométricos observados durante a técnica de trabalho foram: "ruído de topologia" (imperfeições e rugosidades de superfície da imagem), corrigidos pela técnica de smoothing; "presença de chanfro" (arestas pontiagudas), geradas por erros durante a tecelagem do algoritmo no software; "vértice compartilhado" (união de dois vértices em um único 
vértice) gerando união indesejada das estruturas; "gaps" (furos na imagem) gerados por inconsistência durante a rotina de tecelagem; e "auto-interseções" (formação de um volume sólido indesejado em uma superfície oca) geradas por erros durante a tecelagem do algoritmo (Attene et al., 2013).

Figura 3: Imagem que exemplifica a realização programada da técnica de "vértice compartilhado" no software 3d Builder®.

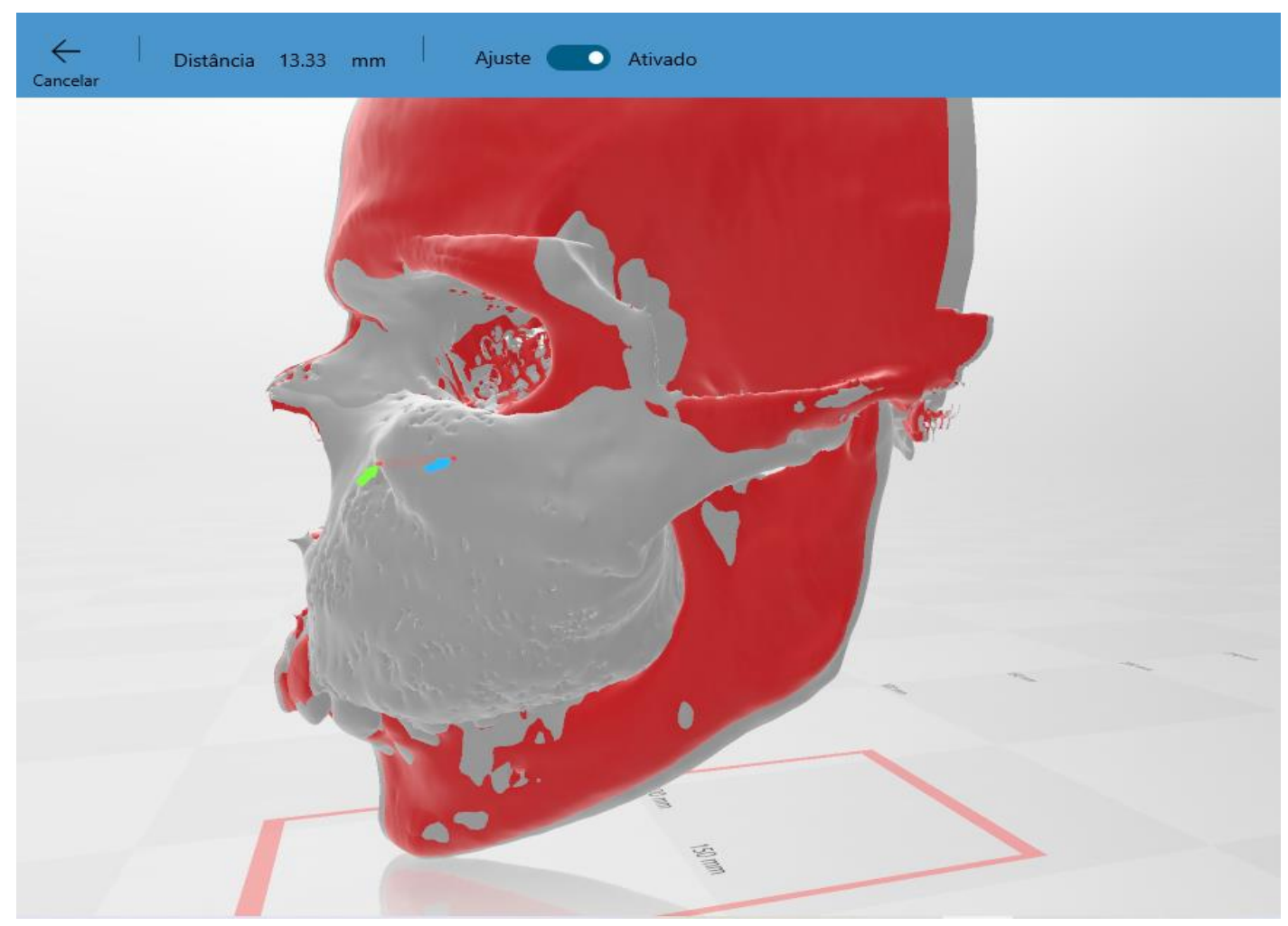

Fonte: Autores.

Para realização desta técnica, houve a segmentação das estruturas faciais na linha média com formação de duas estruturas independentes, pigmentação da peça direita, espelhamento da peça direita e sobreposição aproximada da malha facial direita sobre a esquerda. Esta ferramenta foi utilizada para facilitar a visualização da delimitação e da assimetria gerada pela patologia (em delimitação cinza na imagem). Com as duas faces sobrepostas pode-se utilizar a ferramenta para mensurar as distâncias entre os dois forames infra-orbitários (setas azul e verde). As distâncias entre a seta verde e azul indica a extensão da patologia na região.

A modelagem por FFF é um método de manufatura aditiva incremental no qual a impressora constrói a peça através da fundição da base seletivamente, camada sobre camada, formando um produto único, pré-estabelecido pelo sistema computacional (Stansbury \& Idacavage, 2016). O CPE+ é um copoliéster termoplástico (Co-PolyEster) melhorado com um monómero de base biológica, que se produz por cloração de PET (tereftalato de polietileno- PETG). Este filamento apresenta grande dureza, grande resistência a impactos e a tração. Ao contrário do PET, o CPE+ contém glicol adicionado a sua composição e não exibe cristalização induzida por deformação. Optou-se pelo uso deste material por favorecer a impressão de peças de baixo custo, baixa espessura, translúcidas, resistentes, atóxicas e que apresentam estabilidade dimensional após a esterilização por óxido de etileno (Calvo-Haro et al., 2021). Devido à sua alta tonicidade, transparência e resistência química a utilização do CPE+ é bem estabelecida em aplicações alimentícias, indústrias médicas e eletrônicos. Objetos impressos em CPE+ apresentam moderada resistência a forças laterais (eixo X e Y de impressão) (Roman et al., 2021). Na comparação entre 
o CPE e o PLA (polylactic acid) para FFF, observa-se que o último tem maior resistência e módulo de tração, contudo é menos durável do que o CPE+, pois é confeccionado através de recursos renováveis (como o milho), perdendo propriedades ao longo do tempo (Roman et al., 2021; Sepahi et al., 2021).

A técnica incremental por fatiamento adaptável utilizada em FFF minimizou a distorção térmica associada ao resfriamento não uniforme do feixe do polímero. Ainda, minimizou a chance de erros dimensionais, relacionados principalmente com o eixo de construção $\mathrm{Z}$ (eixo incremental vertical), onde características próprias de dilatação e condensação do filamento, da quantidade e qualidade da estrutura de suporte adicionada ao objeto são mais sensíveis, por estarem distantes da placa de base (Mohan Pandey et al., 2003; Stansbury \& Idacavage, 2016; Zeller et al., 2021).

Após impressão de guia cirúrgica em CPE+ translúcido, através da técnica de FFF, foi possível observar diferença superestimada aproximada de espessura da estrutura de $0,40 \mathrm{~mm}$ quando comparada à mesma estrutura digital (Figura 4A e 4 B). Dados similares foram encontrados por Van den Broeck et al. (2014), através da comparação entre estrutura 3D digital, usando imagens de seu correspondente modelo ósseo de TC, superestimado $0,55 \mathrm{~mm}$ em comparação com o osso real.

Na comparação de volume orbital entre a TC e a subsequente impressão por PR, através do software Solidworks® (Dassault Systèmes S.A, Vélizy-Villacoublay, França) e pela análise direta de preenchimento do protótipo com solução salina, não foram observadas discrepâncias significativas (Roça et al., 2013). Diferenças mínimas, não significativas, também têm sido relatadas na comparação do modelo impresso com peças cadavéricas, o que sugere excelente confiabilidade à técnica de impressão por FFF (Ibrahim et al., 2009; Petropolis et al., 2015; Zeller et al., 2021).

Avaliações geométricas em todo o processo de construção de objetos 3D impressos em impressoras FFF, mostraram que a presença de erro dimensional da imagem 3D reconstruída e do objeto 3D é maior do que a diferença de dimensões entre a imagem adquirida pela tomografia e sua imagem reconstruída. Esses resultados sugerem que a etapa mais suscetível à produção de erros e de distorção da anatomia se dá na etapa pós processamento da imagem DICOM, especialmente na impressão do modelo (Roça et al., 2013). Mesmo que pequenas diferenças e distorções do objeto impresso ocorram, elas não são significativas sob o ponto de vista clínico e cirúrgico, o que apoia o uso dessa tecnologia na área da saúde (Broeck et al., 2014; Fogaça et al., 2011; Kim \& Oh, 2008; Rengier et al., 2010; Roça et al., 2013; Salmi et al., 2013; Silva et al., 2008; Whyms et al., 2013).

A precisão das impressoras 3D pode variar devido a diferentes fatores, sendo altamente dependente do tipo e condicionamento do material disponível para confecção dos biomodelos e disponibilidade e calibragem do equipamento (Ibrahim et al., 2009; Kim et al., 2018; Stansbury \& Idacavage, 2016). 
Figura 4: Imagem que exemplifica a medição em espessura de um guia cirúrgico através do software 3d Builder®.

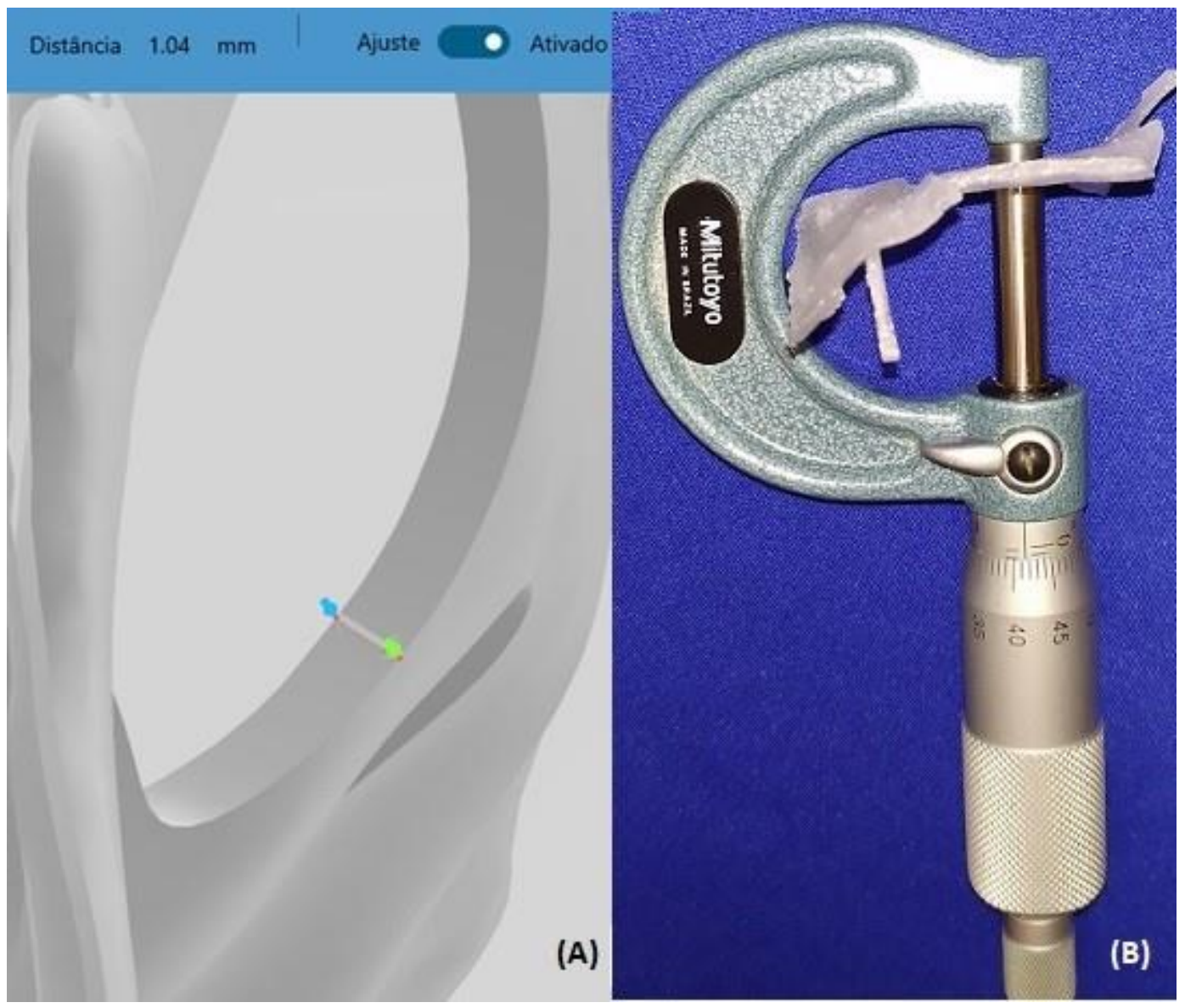

A: Medição digital da espessura $(1,04 \mathrm{~mm})$ do guia cirúrgico confeccionado em software.

B: Medição com especímetro $(1,41 \mathrm{~mm})$ do guia cirúrgico impresso em CPE pela técnica de FFF. Fonte: Autores.

O uso clínico e cirúrgico das diferentes aplicações da PR permite o desenvolvimento de biomodelos com precisão estrutural, baixo custo e rápida confecção. No ato cirúrgico, especialmente envolvendo tecidos duros, os biomodelos servem como ferramentas que facilitam o planejamento e a adaptação dos meios de osteossíntese (placas e parafusos) (Arvier et al., 1994; Assis et al., 2010; D'Urso et al., 1998; Ganry et al., 2018; Ibrahim et al., 2009; Mehrotra \& Markus, 2021; Meurer et al., 2003; Mothes et al., 2018; Silva et al., 2008; Stansbury \& Idacavage, 2016; Steenberghe et al., 2002; Zeller et al., 2021). Por serem praticamente idênticos às estruturas reais, aumentam a previsibilidade cirúrgica, reduzindo o tempo do procedimento e consequentemente diminuem custo e morbidade (Meurer et al., 2003; Mothes et al., 2018; Zeller et al., 2021). Proporcionam aos profissionais o desenvolvimento de uma imagem física e mental tridimensional da região anatômica estudada. Ainda, permitem a alteração do arquivo digital diretamente nos softwares de modelagem para materialização de novo modelo físico, se necessário (Gerstle et al., 2014; Meurer et al., 2003; Stansbury \& Idacavage, 2016; Tramonteno \& Pereira, 2015).

O emprego da PR permite aprendizagem, integração e melhor comunicação entre a equipe assistente e da própria equipe com o paciente. Cada etapa associada a esse grupo de tecnologias, envolve capacitação e oferece incremento na busca de melhorias na assistência à saúde (Zeller et al., 2021).

\section{Considerações Finais}

A utilização da PR na área da saúde é imensurável. O grupo de tecnologias representa um instrumento facilitador, promovendo avanço tecnológico, contribuindo na educação continuada dos profissionais e aprimorando a assistência à saúde. A implementação de novas tecnologias oferece maior acurácia ao diagnóstico e melhora no tratamento cirúrgico buco-maxilo- 
facial, é viável e deve ser estimulada dentro do Sistema Único de Saúde. As etapas de aquisição de imagem, desenvolvimento do estudo digital e a impressão de biomodelos deverão considerar os custos, tempo de confecção, precisão dimensional, qualidade superficial, real aplicabilidade clínica e cirúrgica e disponibilidade de equipamentos e recursos humanos de cada organização hospitalar.

\section{Agradecimentos}

Ao Serviço de Radiologia e Serviço de Pesquisa e Desenvolvimento em Engenharia Biomédica do Hospital de Clínicas de Porto Alegre pela oportunidade e ensinamentos.

\section{Referências}

Arvier, J. F., Barker, T. M., Yau, Y. Y., D'Urso, P. S., Atkinson, R. L., \& McDermant, G. R. (1994). Maxillofacial biomodelling. The British journal of oral \& maxillofacial surgery, 32(5), 276-283.

Assis, G. M., Silva, S. C., Moraes, P., Amaral, J. I., Silva, J. A., \& Germano, A.R. (2010). Auxílio da prototipagem na reconstrução mandibular: caso clínico. Rev. Cir. Traumatol. Buco-Maxilo-Fac., Camaragibe, 10, 3.

Attene, M., Campen, M., \& Kobbelt, L. P. (2013). Polygon mesh repairing: An application perspective. ACM Comput. Surv., 45, 15:1-15:33.

Bagaria, V., \& Chaudhary, K. (2017). A paradigm shift in surgical planning and simulation using 3Dgraphy: Experience of first 50 surgeries done using 3Dprinted biomodels. Injury, 48(11), 2501-2508.

Calvo-Haro, J. A., Pascau, J., Asencio-Pascual, J. M., Calvo-Manuel, F., Cancho-Gil, M. J., Del Cañizo López, J. F., Fanjul-Gómez, M., García-Leal, R., González-Casaurrán, G., González-Leyte, M., León-Luis, J. A., Mediavilla-Santos, L., Ochandiano-Caicoya, S., Pérez-Caballero, R., Ribed-Sánchez, A., RíoGómez, J., Sánchez-Pérez, E., Serrano-Andreu, J., Tousidonis-Rial, M., Vaquero-Martín, J., \& Perez-Mañanes, R. (2021). Point-of-care manufacturing: a single university hospital's initial experience. $3 D$ printing in medicine, 7(1), 11.

Dalmazo, J., Elias Júnior, J., Brocchi, M. A. C., Costa, P. R., \& Azevedo-Marques, P. M. de. (2010). Otimização da dose em exames de rotina em tomografia computadorizada: estudo de viabilidade em um Hospital Universitário. Radiologia Brasileira, 43(4), $241-248$.

Dovales, A. C. M., de Souza, A. A., \& Veiga, L. H. S. (2016). Tomografia computadorizada no Brasil: frequência e padrão de uso em pacientes internados no Sistema Único de Saúde (SUS). Revista Brasileira De Física Médica, 9(1), 11-14.

D'Urso, P. S., Atkinson, R. L., Lanigan, M. W., Earwaker, W. J., Bruce, I. J., Holmes, A., Barker, T. M., Effeney, D. J., \& Thompson, R. G. (1998). Stereolithographic (SL) biomodelling in craniofacial surgery. British journal of plastic surgery, 51(7), 522-530.

Eijnatten, M. van, van Dijk, R., Dobbe, J., Streekstra, G., Koivisto, J., \& Wolff, J. (2018). CT image segmentation methods for bone used in medical additive manufacturing. Medical engineering \& physics, 51, 6-16.

Fogaça, A., Dreyer, J. W., Júnior, A. N., Moraes, J. F., \& Oliveira, M. G. (2011). Dimensional accuracy of select laser sintering and three-dimensional printing biomodels after autoclaving: a preliminary descriptive study. Archives of Oral Research, 7(1), 27-33.

Ganry, L., Hersant, B., Bosc, R., Leyder, P., Quilichini, J., \& Meningaud, J. P. (2018). Study of medical education in 3D surgical modeling by surgeons with free open-source software: Example of mandibular reconstruction with fibula free flap and creation of its surgical guides. Journal of stomatology, oral and maxillofacial surgery, 119(4), 262-267.

Gerstle, T. L., Ibrahim, A., Kim, P. S., Lee, B. T., \& Lin, S. J. (2014). A plastic surgery application in evolution: three-dimensional printing. Plastic and Reconstructive Surgery, 133(2), 446-451.

Ibrahim, D., Broilo, T. L., Heitz, C., de Oliveira, M. G., de Oliveira, H. W., Nobre, S. M., Dos Santos Filho, J. H., \& Silva, D. N. (2009). Dimensional error of selective laser sintering, three-dimensional printing and PolyJet models in the reproduction of mandibular anatomy. Journal of Cranio-Maxillo-Facial Surgery: official publication of the European Association for Cranio-Maxillo-Facial Surgery, 37(3), 167-173.

Jacobs, S., Grunert, R., Mohr, F. W., \& Falk, V. (2008). 3D-Imaging of cardiac structures using 3D heart models for planning in heart surgery: a preliminary study. Interactive Cardiovascular and Thoracic Surgery, 7(1), 6-9.

Ju, T. (2009) Fixing Geometric Errors on Polygonal Models: A Survey. J. Comput. Sci. Technol. 24, 19-29.

Kim, G., \& Oh, Y.T. (2008). A benchmark study on rapid prototyping processes and machines: Quantitative comparisons of mechanical properties, accuracy, roughness, speed, and material cost. Proceedings of the Institution of Mechanical Engineers, Part B: Journal of Engineering Manufacture, 222,201 - 215.

Kim, S. Y., Shin, Y. S., Jung, H. D., Hwang, C. J., Baik, H. S., \& Cha, J. Y. (2018). Precision and trueness of dental models manufactured with different 3dimensional printing techniques. American Journal of Orthodontics and Dentofacial Orthopedics: official publication of the American Association of Orthodontists, its constituent societies, and the American Board of Orthodontics, 153(1), 144-153.

Lechuga, L., \& Weidlich, G. A. (2016). Cone Beam CT vs. Fan Beam CT: A Comparison of Image Quality and Dose Delivered Between Two Differing CT Imaging Modalities. Cureus, 8(9), e778. 
Li, Z., Li, Z., Xu, R., Li, M., Li, J., Liu, Y., Sui, D., Zhang, W., \& Chen, Z. (2015). Three-dimensional printing models improve understanding of spinal fracture--A randomized controlled study in China. Scientific reports, 5, 11570.

Mehrotra, D., \& Markus, A. F. (2021). Emerging simulation technologies in global craniofacial surgical training. Journal of Oral Biology and Craniofacial Research, 11(4), 486-499.

Meshmixer. (2021). Autodesk. https://www.meshmixer.com/

Meurer, E., Oliveira, M. G., Meurer, M. I., Silva, J. V. L., Bárbara, A. S., \& Heitz C. (2003). Biomodelos de Prototipagem Rápida em CTBMF. Rev. Bras. Cir. Periodontia, Curitiba, 1(3), 172-180.

Meurer, M. I., Meurer, E., Silva, J. V. L., Bárbara A. S., Nobre, L. F., Oliveira, M. G., \& Silva, D.M. (2008). Aquisição e manipulação de imagens por tomografia computadorizada da região maxilofacial visando à obtenção de protótipos biomédicos. Radiologia Brasileira [online]. 41 (1), $49-54$.

Mitsouras, D., Liacouras, P., Imanzadeh, A., Giannopoulos, A. A., Cai, T., Kumamaru, K. K., George, E., Wake, N., Caterson, E. J., Pomahac, B., Ho, V. B., Grant, G. T., \& Rybicki, F. J. (2015). Medical 3D Printing for the Radiologist. Radiographics: a review publication of the Radiological Society of North America, Inc, 35(7), 1965-1988.

Mohan Pandey, P., Venkata Reddy, N., \& Dhande, S. G. (2003). Slicing procedures in layered manufacturing: a review. Rapid Prototyping Journal, 9(5), 274288.

Mothes, F. C., Britto, A., Matsumoto, F., Tonding, M., \& Ruaro, R. (2018). Application of three-dimensional prototyping in planning the treatment of proximal humerus bone deformities. Revista Brasileira de Ortopedia, 53(5), 595-601.

Oliveira, A. I. A. de, Assis, G. J. A. de, \& Garotti, M. F. (2014). Tecnologias no ensino de crianças com paralisia cerebral. Revista Brasileira de Educação Especial, 20(1), 85-102.

Petropolis, C., Kozan, D., \& Sigurdson, L. (2015). Accuracy of medical models made by consumer-grade fused deposition modelling printers. Plastic surgery (Oakville, Ont.), 23(2), 91-94.

Puricelli, E. (2014). Técnica anestésica, exodontia e cirurgia dentoalveolar-Série ABENO-Odontologia Essencial-Parte clínica. Porto Alegre: Editora Artes Medicas.

Rengier, F., Mehndiratta, A., von Tengg-Kobligk, H., Zechmann, C. M., Unterhinninghofen, R., Kauczor, H. U., \& Giesel, F. L. (2010). 3D printing based on imaging data: review of medical applications. International Journal of Computer Assisted Radiology and Surgery, 5(4), 335-341.

Roça, G. B., Foggiatto, J. A., Ono, M. C., Ono, S. E., \& da Silva Freitas, R. (2013). Comparison of orbital volume obtained by tomography and rapid prototyping. The Journal of craniofacial surgery, 24(6), 1877-1881.

Roman, N., Cojocaru, D., Coman, C., Repanovici, A., Bou, S. F., \& Miclaus, R. S. (2021). Materials for Respiratory Masks in the Context of COVID 19 Pandemic. Materiale Plastice, 57(4), 236-247.

Salmi, M., Paloheimo, K. S., Tuomi, J., Wolff, J., \& Mäkitie, A. (2013). Accuracy of medical models made by additive manufacturing (rapid manufacturing). Journal of Cranio-maxillo-facial surgery: official publication of the European Association for Cranio-Maxillo-Facial Surgery, 41 (7), 603609.

Sepahi, M. T., Abusalma, H., Jovanovic, V., \& Eisazadeh, H. (2021). Mechanical Properties of 3D-Printed Parts Made of Polyethylene Terephthalate Glycol. Journal of Materials Engineering and Performance, 30(9), 6851-6861.

Shui, W., Zhou, M., Chen, S., Pan, Z., Deng, Q., Yao, Y., Pan, H., He, T., \& Wang, X. (2017). The production of digital and printed resources from multiple modalities using visualization and three-dimensional printing techniques. International Journal of Computer Assisted Radiology and Surgery, 12(1), 13-23.

Silva, D. N., Gerhardt de Oliveira, M., Meurer, E., Meurer, M. I., Lopes da Silva, J. V., \& Santa-Bárbara, A. (2008). Dimensional error in selective laser sintering and 3D-printing of models for craniomaxillary anatomy reconstruction. Journal of cranio-maxillo-facial surgery: official publication of the European Association for Cranio-Maxillo-Facial Surgery, 36(8), 443-449.

Stansbury, J. W., \& Idacavage, M. J. (2016). 3D printing with polymers: Challenges among expanding options and opportunities. Dental Materials: official publication of the Academy of Dental Materials, 32(1), 54-64.

Steenberghe, D. van, Naert, I., Andersson, M., Brajnovic, I., Cleynenbreugel, J. van, \& Suetens, P. (2002). A custom template and definitive prosthesis allowing immediate implant loading in the maxilla: a clinical report. The International Journal of Oral \& Maxillofacial Implants, 17(5), 663-670.

Tramontano, M., \& Junior, A. P. (2015). Ressignificando o modelo físico: impressão 3D e ensino de projeto de arquitetura. Anais do XIX Congresso Da Sociedade Ibero-Americana de Gráfica Digital 2015.

Van den Broeck, J., Vereecke, E., Wirix-Speetjens, R., \& Vander Sloten, J. (2014). Segmentation accuracy of long bones. Medical engineering \& physics, 36(7), 949-953.

Volpato, N. (2007). Integração da prototipagem rápida com o processo de desenvolvimento de produto. In: Prototipagem rápida: tecnologias e aplicações. Edgard Blucher.

Whyms, B. J., Vorperian, H. K., Gentry, L. R., Schimek, E. M., Bersu, E. T., \& Chung, M. K. (2013). The effect of computed tomographic scanner parameters and 3-dimensional volume rendering techniques on the accuracy of linear, angular, and volumetric measurements of the mandible. Oral surgery, Oral Medicine, Oral Pathology and Oral Radiology, 115(5), 682-691.

Zeller, A. N., Neuhaus, M. T., Fresenborg, S., Zimmerer, R. M., Jehn, P., Spalthoff, S., Gellrich, N. C., \& Dittmann, J. A. (2021). Accurate and cost-effective mandibular biomodels: a standardized evaluation of 3D-Printing via fused layer deposition modeling on soluble support structures. Journal of Stomatology, Oral and Maxillofacial Surgery, 122(4), 355-360. 